\title{
Equilibrium structure of decagonal AlNiCo
}

\author{
S. Naidu, M. Mihalkovič ${ }^{1}$ and M. Widom, \\ Department of Physics, Carnegie Mellon University, Pittsburgh, PA 15213 \\ ${ }^{1}$ also at: Institute of Physics, Slovak Academy of Sciences, 84228 Bratislava, Slovakia
}

\begin{abstract}
We investigate the high temperature decagonal quasicrystalline phase of $\mathrm{Al}_{72} \mathrm{Ni}_{20} \mathrm{Co}_{8}$ using a quasilattice gas Monte-Carlo simulation. To avoid biasing towards a specific model we use an over-dense site list with a large fraction of free sites, permitting the simulation to explore an extended region of perpendicular space. Representing the atomic surface occupancy in a basis of harmonic functions directly reveals the 5 -fold symmetric component of our data. Occupancy is examined in physical and perpendicular space.
\end{abstract}

\section{INTRODUCTION}

AlNiCo exhibits quasicrystalline phases over a range of compositions and temperatures[1]. Of special interest is the Ni-rich quasicrystalline phase around the composition $\mathrm{Al}_{72} \mathrm{Ni}_{20} \mathrm{Co}_{8}$. This is a decagonal phase with a period of $4.08 \AA$ along the periodic axis, making it a simple phase relative to other members of the Al-Ni-Co family. Additionally, it appears to be most perfect structurally, even though it is stable only at high temperatures around $\mathrm{T}=1000-1200 \mathrm{~K}$. Its structure has been extensively studied experimentally by X-ray diffraction $[2,3]$ and electron microscopy $[4,5]$. Finally, since qualitatively accurate pair potentials are available[6], structural predictions can be made based on total energy considerations[7].

An idealized deterministic structure for this phase has been proposed by studying the total energy[7]. This prediction employed a multi-scale simulation method, in which small system sizes were simulated starting with very limited experimental input, then rules discovered through the small scale simulations were imposed to accelerate simulations of larger-scale models. Although efficient, this approach leaves open the question of how strongly the final model was biased by the chosen method.

We adopt a different approach here, starting from slightly different experimental input and working directly at the relevant high temperatures. The experimental input is: (1) the density [2], composition and temperature at which the phase exists; (2) the hyperspace positions of atomic surfaces (these are simply the positions for a Penrose tiling, with AS1 at $\nu= \pm 1$ and AS2 at $\nu= \pm 2$ ); (3) the fact that the quasicrystal is layered, with space group $10_{5} / m m c$, and its lattice constants (we take $a_{q}=6.427, c=4.08 \AA$ ). The chief unknowns to be determined are the sizes, shapes and chemical occupancies of the atomic surfaces.

Like the prior study [7], we employ Monte Carlo simulation using the same electronic-structure derived pair potentials[6]. Also, like the prior study, we employ a discrete list of allowed atomic positions. However, instead of using sparse decorations of fundamental tiles, where the configurational freedom arose largely from flipping of the decorated tiles, in the present study we employ a fixed site list based on a rich decoration of fixed tiles. For a given tiling the density of allowed sites in our new simulation is much greater than the actual atomic density. The resulting atomic surface (see Fig. 1a) corresponds to that of a Penrose tiling with tile edge length $a_{q} / \tau^{3}=1.52 \AA$ (plus a few additional sites such as inside some fat rhombi). These atomic surfaces include within them the atomic surfaces previously proposed on the basis of total energy calculations [7], 
(a)

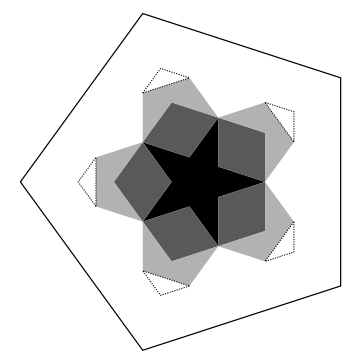

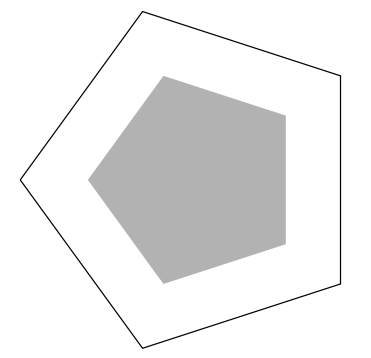

(b)
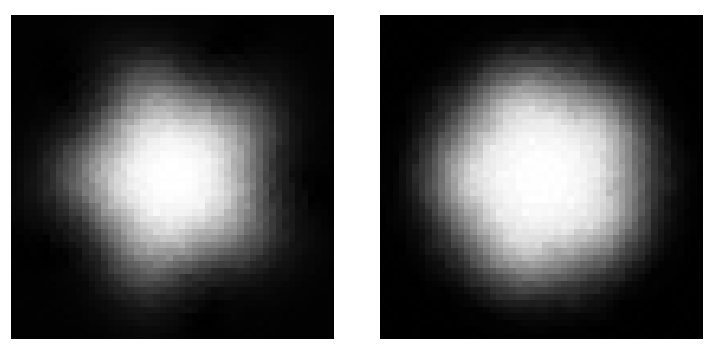

Figure 1: (a) Oversized atomic surface used in the present simulation. AS1 (left) at $\nu= \pm 1$, AS2 (right) at $\nu= \pm 2$. For comparison the ideal model proposed in Ref. [7] is inscribed inside. Color scheme: black $=\mathrm{Co}$, gray $=\mathrm{Ni}$, light-gray $=\mathrm{Al}$, white= $=$ partial $\mathrm{Al}$ occupancy. (b) Total occupancy of AS1 (left) and AS2 (right) at T=1160K.

and analysis of experimental data $[2,3]$. This larger atomic surface avoids any bias towards particular atomic surface shapes.

The following section describes the site list and other simulation details more precisely. Then we present site occupancy in real space and on the atomic surfaces. We represent atomic surface occupancies using Fourier-Bessel series, allowing us to isolate the five-fold symmetric component and filter sampling noise. Such a representation could prove fruitful in crystallographic structure determination as well. Refs. [8, 9] pursued related goals.

We compare our results with a recent experimental study. Briefly, we find broad agreement with the sizes, shapes and chemical occupancies of the atomic surfaces. A few specific points of disagreement can be addressed by relaxing our fixed site positions. Finally, we conclude with a summary of our results and outlook for future work.

\section{SIMULATION METHOD}

We should perform simulations in the continuum, with no predefined site list to bias our results. Unfortunately, strong binding of Al to transition metal atoms (Co and $\mathrm{Ni}$ ) leads to phase separation into a CsCl-type crystal plus regions of excess pure Al. This reflects a deficiency of our interatomic potentials, which should not be applied at a transition-metal concentration as high as the $50 \%$ found in CsCl-type crystals. Restricting atomic positions a set that does not include the $\mathrm{CsCl}$ crystal inhibits phase separation.

Rather than specify these sites in a complex manner whose details may fail to allow some especially favorable structure, we take a relatively neutral assignment that still allows quasicrystalline structures to form. Specifically we restrict the possible atomic positions to the vertices of a Penrose lattice whose edge length is small compared to the spacing between atoms. The possible atomic sites fill space with a density 2.55 times greater than the actual atomic density, so the majority of sites are empty in any particular configuration. Our prior study of prefered structures proposed an idealized arrangement of atoms on an HBS (hexagon-boat-star) tiling of edge length $a_{q}$, as illustrated in Fig. 2.

Our Monte Carlo simulation distributes atoms among these sites in a manner consistent with thermal equilibrium at a given temperature. We focus on the temperature $\mathrm{T}=1160 \mathrm{~K}$, which lies within the stability range for the Ni-rich decagonal phase [1]. We simulate a low phason strain quasicrystal approximant, with lattice parameters 

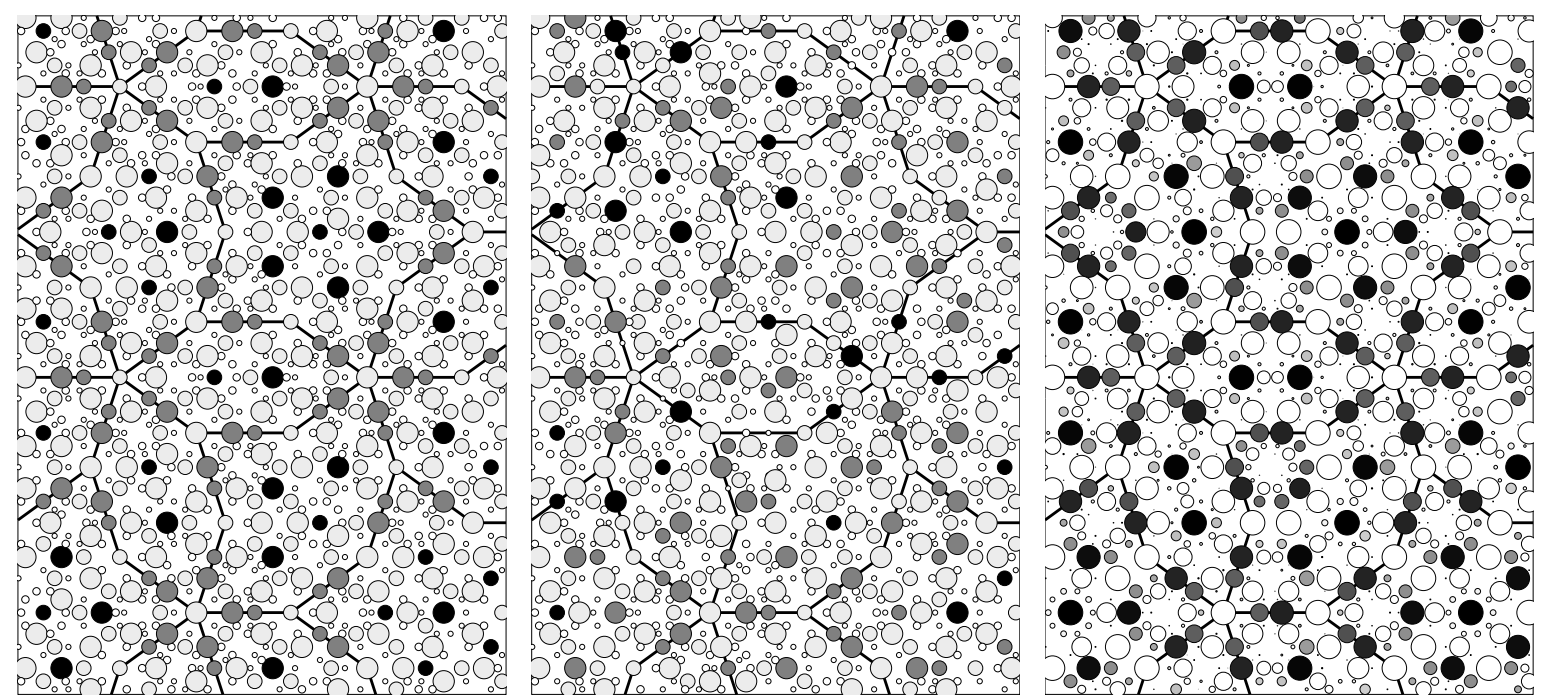

Figure 2: This figure illustrates our fixed site list in parallel space. Ideal tile decoration (left) and typical $\mathrm{T}=1160 \mathrm{~K}$ configuration (center). Color scheme as in Fig 1a. Large/small atoms indicate upper/lower layer. Very small white circles indicate vacancies. Occupancy data (right) at $\mathrm{T}=1160 \mathrm{~K}$ with atomic size indicating total occupancy and $\mathrm{Al}$ fraction indicated by gray scale (black=100\% TM).

$159.38 \times 83.79 \times 4.08 \AA^{3}$, containing 9776 lattice sites and holding 3838 atoms. We initially decorated the structure randomly at the desired density and composition. We then lowered the temperature in steps from an initial $\mathrm{T}=4000 \mathrm{~K}$, annealing at each temperature, until we reached our data collection temperature of $\mathrm{T}=1160 \mathrm{~K}$.

Our data analysis differs from conventional approaches to quasicrystal atomic surface modeling. It is common to model atomic surface occupancy by breaking the atomic surface up into polygonal domains piecewise constant occupancy in each domain. This approach highlights the manner in which occupancy depends on the local real space environment, which changes discontinuously as a function in perpendicular space. Unfortunately such a piecewise continuous representation does not properly capture the effects of chemical disorder and phason fluctuations characteristic of a quasicrystal at high temperatures.

We show below that the mean occupancy statistics actually vary quite smoothly over the atomic surface. To represent smoothly varying atomic surface occupancy functions it is helpful to introduce a complete basis set of analytic functions and expand the occupancy in this basis. Owing to the axial symmetry of the atomic surfaces it is handy to introduce polar coordinates $\left(r_{\perp}, \theta\right)$. The natural function basis set for polar coordinates are combinations of cylindrical Bessel functions multiplied by complex exponentials. Thus, on a given atomic surface we express the occupancy by chemical species $\alpha$ as

$$
\rho_{\alpha}\left(r_{\perp}, \theta\right)=\sum_{m, n} A_{m, n}^{\alpha} J_{m}\left(k_{m, n} r_{\perp}\right) e^{i m \theta} .
$$

The $m$ index represents the angular mode frequency. For a given angular frequency $m$, the sum over $n$ allows the representation of an arbitrary radial variation, with radial frequencies $k_{m, n}$ such that all Bessel functions share a common zero outside our atomic surface. To analyze our data we invert eq. (1) to obtain the coefficients $A_{m, n}^{\alpha}$ using orthonormality of the basis set. We then filter out the components whose angular variation lacks 5 -fold symmetry by restricting $m$ to multiples of 5 . 

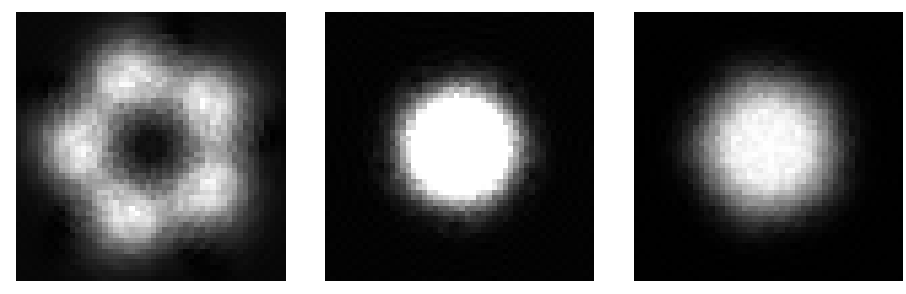

Figure 3: This figure illustrates the chemical occupancy of the $\nu= \pm 1$ atomic surfaces for $\mathrm{Al}, \mathrm{Ni}$ and Co atoms (left to right). The gray scales are proportional to occupancy, scaled to the maximum for each species.

\section{RESULTS}

Each Monte Carlo step consisted of many attempted jumps (of up to $6.5 \AA$ in length) per atom. An attempted jump to an occupied site means an attempt to swap the two atoms. We recorded the detailed atomic site occupancy for 9000 configurations separated by sufficiently many Monte Carlo steps that the configurations become uncorrelated.

Phason fluctuations such as bowtie flips [7] cause the center of gravity of the structure to drift in perpendicular space. Note that the lattice sites do not move during a phason flip, only their atomic occupancy changes. A bowtie flip in our ideal model interchanges two NiNi pairs with two AlCo pairs. To avoid smearing caused by this drift, which could obscure small-scale atomic surface details, we recentered the perp space center of gravity for each configuration before projecting onto the Fourier-Bessel coefficients. Without recentering the atomic surface occupancy functions would be weaker and broader.

As in our prior [7] low temperature study, we find that the HBS tiling effectively describes the quasiperiodic layers, but the prefered chemical decoration of the tiles now becomes highly variable at certain sites. Fig. 1(b) displays the average occupancy distribution on each atomic surface. The AS1 occupancy is a combination of all three chemical species. The AS2 occupancy is virtually 100\% Al. The breakdown of AS1 occupancy among $\mathrm{Al}, \mathrm{Ni}$ and $\mathrm{Co}$ is shown in Fig. 3. A striking feature of all these occupancy plots is the smooth, continuous variation of occupancy. It is clearly not appropriate to model these densities using piecewise constant functions with polygonal boundaries, even though five-fold symmetry is present. Ni and Co are strongly mixed with very similar distributions, concentrated at the center of AS1. Because the amount of Ni present exceeds the amount of $\mathrm{Co}$, the Co occupancy vanishes more quickly than the $\mathrm{Ni}$ occupancy away from the center, while the Ni occupancy remains more nearly constant. In the region where the TM occupancy drops off it is largely replaced with $\mathrm{Al}$ so the total occupancy remains close to $100 \%$ in this crossover region.

In parallel space atoms in the TM to $\mathrm{Al}$ crossover region correspond to tile edges. Effects of this gradient from TM to Al occupancy can be seen in Fig. 2 (right), near tiling vertices at which two or more edges join in a $\pm 72^{\circ}$ angle. The pair of occupied sites on these tile edges show unequal Al/TM occupancy. The site closer to the vertex contains relatively less TM and more $\mathrm{Al}$ than the site further away. The site closer to the vertex in parallel space lies in the atomic surface region that is further from the center in perp space. Note also that bowtie phason flips that replace TM-TM pairs with Al-TM pairs on tile edges are one type of excitation that replace the TM site close to a $72^{\circ}$ vertex with an $\mathrm{Al}$ atom. We understand these effects energetically because the inner edge site is in a TM-rich environment. Al-TM bonds are strongly favorable so this is a likely site for Al occupancy.

Far from the atomic surface centers, where the occupancy falls smoothly to zero, the 
atoms are mainly Al. These highly mobile atoms are in locations in parallel space where there are multiple ideal sites, too close to be simultaneously occupied, but each one with a rather similar local environment so that there is no strong energetic preference among the sites, for example the pair of symmetry-related sites near the center of the hexagon tile. Other examples are rings of 10 sites surrounding the ideal Co position at the centers of boat and star tiles. These rings can hold at most three $\mathrm{Al}$ atoms. Jumps of $\mathrm{Al}$ atoms among these sites correspond to phason tile flips of the underlying very small $1.52 \AA$ rhombus tiling.

\section{COMPARISON WITH EXPERIMENT}

We compare primarily with the refinement by Takakura [2]. We first point areas of general agreement between our results and the experimental data, then discuss the main points of disagreement. Our simulated occupation probabilities generally agree well with experiment. The approximate sizes and shapes of our atomic surfaces agree with Takakura. We agree that AS1 contains transition metal atoms within a central region (Takakura orbit numbers 1-5), separated by a fully occupied region of mixed Al/TM (orbits 6 and 7) and finally radial spokes in which $\mathrm{Al}$ occupancy diminishes from full to partial (orbits 8-10). We agree that AS2 (orbit numbers 11-23) contains primarily Al atoms. Takakura finds full occupancy of orbit numbers 14 and 16, but only $82 \%$ occupancy of orbit numbers 15 and 17, which are locally fairly similar (these are the Al sites adjacent to tile edge TM atoms). Our results qualitatively support this, with occupancy about $90 \%$ corresponding to orbit numbers 14 and 16, dropping to around 70-80\% for orbit numbers 15 and 17.

Now consider discrepancies between our results and experiment. Orbits 19 and 23, localized around special points on the fringes of AS2 are fully occupied in the refinement, while we find partial occupancy. In parallel space these correspond to pairs of ideal sites midway between TM atoms. Partial occupancy is forced because the spatial separation of these ideal points $(2.25 \AA)$ is too small to allow both to be simultaneously occupied. However, if we consider multilayer strucures, with $c=4 \times 4.08 \AA$, and allow structural relaxation, we know [10] it is possible to occupy 3 out of every 4 such sites per $8.16 \AA$. The lack of structural relaxation thus causes an error in our occupancy for this orbit.

Takakura's fully occupied orbits numbers 20-22 are located on the corners of AS2. We find $60-80 \%$ occupancy instead of full occupancy, consistently with our smoothly decreasing occupation probability. Takakura also finds a small fraction (about 20\%) of TM atoms on orbits 20 and 21, while we find only Al. There is no strong energetic reason that TM atoms should not occupy those sites. Perhaps the TM occupancy of orbit number 20 is related to the large atomic displacement of this orbit Takakura found during the refinement.

Surprisingly, Takakura finds $50 \%$ occupancy on orbit number 11 , at the very center of AS2. In parallel space this site corresponds to HBS tile vertices at which five edges meet. We find it is fully occupied, similar to all other HBS tile vertices.

\section{CONCLUSION}

We presented a method to compute atomic surface occupancy statistics, and applied it to the basic Ni-rich decagonal phase of AlNiCo. This work launches a promising set of possible future research directions. The method can be applied to other decagonal phases and also to icosahedral phases. The main limitation is the availability of interatomic

potentials. Further, a great deal remains to be done even within AlNiCo, as we now discuss.

Our simulations generate a complete set of equilibrium configurations, in which the precise location of every atom is known. The analysis above only presents the atomic 
surface occupation probabilities, but does not address correlations among the partially occupied sites. Such correlations are contained in the Patterson function, which is also known experimentally [11] and can be computed from our existing data. Actual atomic configurations contain more information than the atomic surface occupancies, because all correlations are reflected in the actual configurations. Fig. 2 (center) illustrates an atomic arrangement from our simulation. Comparing with the ideal model (left) reveals a great deal of disorder, including phason flips, chemical disorder and vacancies.

Among the prominent correlations are strong anticorrelation of too-close sites. The deep clefts between arms of AS1 correspond to sites that are close in parallel space to sites near the corners of AS2. When these AS2 sites are occupied sites within the clefts of AS1 are empty. When these AS2 sites are empty then we find partial occupancy inside the clefts of AS1. Another anticorrelation occurs because the pairs of ideal sites midway between pairs of TM atoms (e.g. Takakura orbit number 19 and 23) are too close for simultaneous occupancy. This anticorrelation arises because we used only "ideal" positions projected into parallel space from flat atomic surfaces. In reality the atomic positions are displaced somewhat away from the ideal sites, and we showed above that such displacements can have a substantial influence on occupancy statistics in cases where the ideal sites are just slightly too close together. Indeed, relaxations of our ideal structures, using either pair potentials [10] or full ab-initio calculations result in displacements similar to those reported experimentally [2].

Finally, our simulation used only a single $4.08 \AA$ repeat unit along the 10 -fold symmetry axis. Owing to our periodic boundary conditions, swapping a pair of atoms in the single repeat implies a simultaneous swap of all pairs of atoms in infinite columns extending above and below the original pair in our single repeat. But when we calculate the energy for the swap, we only include the energies of the original atoms. This type of finite-size error needs to be eliminated, as it miscalculates the energies of phason flips.

Simply stacking two repeats above each other to make an $8.16 \AA$ unit, however, usually overestimates the energy cost of atom swaps corresponding to bowtie phason flips. This is because uncorrelated flips between repeat units create phason stacking faults whose energy cost is large in the absence of atomic relaxation but is small, and can even be negative, when relaxation is included. We must include relaxed atomic positions to achieve a realistic model of three-dimensional phason disorder.

\section{ACKNOWLEDGEMENTS}

We acknowledge discussions with C.L. Henley support by NSF grant DMR-0111198.

\section{REFERENCES}

1. S. Ritsch, C. Beeli et al., Phil. Mag. Lett, 78, 67 (1998).

2. H. Takakura, A. Yamamoto and A.-P. Tsai, Acta Cryst. A, 57, 576 (2001).

3. A. Cervellino, T. Haibach and W. Steurer, Acta Cryst. B, 58, 8 (2002).

4. E. Abe, K. Saitoh et al., Phys. Rev. Lett., 84, 4609 (2000).

5. Y. Yan, S. J. Pennycook and A.-P. Tsai, Phys. Rev. Lett., 81, 5145 (1998).

6. I. Al-Lehyani et al., Phys. Rev. B, 64, 075109 (2001).

7. M. Mihalkovič et al., Phys. Rev. B, 65, 104205 (2002).

8. L. Elcoro and J. M. Perez-Mato and G. Madaraiga, Acta Cryst. A, 50, 182 (1994).

9. M. V. Jaric and S.-Y. Qiu, Phys. Rev. B, 49, 6614 (1994).

10. C. L. Henley, M. Mihalkovič and M. Widom, J. Alloys and Comp., 342, 221 (2002).

11. W. Steurer, T. Haibach, B. Zhang, S. Kek and R. Luck, Acta Cryst. B, 49, 66, (1993). 